\title{
Family socioeconomic status and nutrition habits of 7-8 year old children: cross-sectional Lithuanian COSI study
}

\author{
Aušra Petrauskienè, Vilma Žaltauské ${ }^{*}$ and Edita Albavičiūtè
}

\begin{abstract}
Background: Nutritional habits are a useful way to characterize whole diets and they are also known to be influenced by a wide range of social and economic factors. The above factors in each country may have different effect on children's eating habits. In Lithuania the data of children nutrition in association with socio-economic status of family is poor. There are few studies done, where links between nutrition habits of children and socio-economic status of family was evaluated. The aim of this paper is to evaluate association among nutrition habits of first-formers and family socio-economic status in Lithuania.
\end{abstract}

Methods: Data were obtained participating in the international study, which was performed in all ten districts of Lithuania. A cross-sectional study was carried out in 2010, using the protocol and methodology prepared by the experts from the $\mathrm{WHO}$ and countries participating in the Initiative. The data were collected by means of COSI standardized questionnaire, which was filled out by parents of selected first-formers'. In this paper a part of questions regarding children nutrition habits and parents' socio-economic status is presented. Statistical analysis was performed by using SPSS 20.0 software for Windows. Correlation among variables was evaluated by $x^{2}$. Links among nutrition habits of first-formers and family socioeconomic status were determined using binary logistic regression to calculate odds ratios (OR) and 95\% confidence intervals $(\mathrm{Cl})$. For all tests $p<0.05$ was considered significant.

Results: It was established that the majority (76\%) of Lithuanian first-formers eat breakfast every day or 4-6 times a week. Significant differences were found between breakfast consumption and gender - girls eat breakfast less frequently than boys. Odds ratio of children daily breakfast consumption were 1.3 times higher in families where fathers' were older than 30 years comparing with younger fathers. Meanwhile mothers' age had significant influence just on children daily soft drinks with sugar consumption.

Conclusions: Results from the national survey of primary school age children of Lithuania reveals that family socio-economic position plays one of the major role in breakfast, fresh fruit and soft drinks with sugar consumption among younger school age children.

Keywords: Children, Nutrition habits, Socioeconomic status

\section{Introduction}

Healthy diet of children is associated with positive affect on children growth, development of musculoskeletal, cardiovascular and mental health [1-3]. Nutritional habits are a useful way to characterize whole diets and they are also known to be influenced by a wide range of social and economic factors [4-8]. The above factors in

\footnotetext{
* Correspondence: vilmazaltauske@gmail.com

Institute of Health Research, Academy of Medicine, Lithuanian University of Health Sciences, Kaunas, Lithuania
}

(c) 2015 Petrauskienè et al.; licensee BioMed Central. This is an Open Access article distributed under the terms of the Creative

each country may have different effect on children's eating habits. Some of the research show that children and adolescents from low income families tend to consume less fruit and vegetables and more sugar, fats, processed meat, soft drinks, salty snacks compared with those from higher social class households [9-15]. According to researchers the causal mechanisms may be that healthy foods are frequently more expensive, families in poverty live options or families on lower incomes differ in terms of their education and food culture, leading them to 
make less healthy food choices in areas with lower availability of healthy food $[9,15]$. Therefore understanding these factors is of great importance for the development of relevant new health policies, programmes, and interventions. A new WHO (World Health Organization) policy for health - Health 2020: the European Policy for Health and Well-being is seeking to stop growing social and health inequalities in Europe. One of strategic goals of Lithuania participating in the policy is to develop healthy eating habits [16]. In Lithuania the data of children nutrition in association with socio-economic status of family is poor. There are few studies done, where links between nutrition habits of children and socio-economic status of family was evaluated [17]. There is a need to explore and better understand the key determinants of children nutritional habits within a socio-economic framework in Lithuania. The aim of this paper is to evaluate association among nutrition habits of first-formers and family socio-economic status in Lithuania.

\section{Material and methods}

WHO Regional Office for Europe established a European childhood obesity surveillance system (COSI). Data were obtained participating in the international study, which was performed in all ten districts of Lithuania. A cross-sectional study was carried out in 2010, using the protocol and methodology prepared by the experts from the $\mathrm{WHO}$ and countries participating in the Initiative. A multilevel sampling method (district, school and class) was employed for composing a nationally representative sample (6). The sample size in each of all ten districts of the country was calculated following the data from the Department of Statistics of Lithuania about the number of targeted children. Schools were randomly selected from the list of Ministry of Education and Science. In the 57 municipalities 155 schools were selected for the survey. First class was chosen as a sampling unit.

\section{Questionnaire}

The data were collected by means of COSI standardised questionnaire, which was filled out by parents of selected first-formers'. The optional items of the questionnaire involved the information on children lifestyle and their families' characteristics. In this paper a part of questions regarding children nutrition habits and parents' socioeconomic status is presented. The questions concerning habitual food consumption has not been validated for estimating total intakes of energy or nutrients but is appropriated for exploring dietary patterns on the basis of frequencies. The alternative frequencies for food and drink items and meal patterns were 'never', 'once or twice per month, ' 1 - 3 times a week, ' $4-6$ times a week', and 'every day'. Socio-economic characteristics of family were evaluated by parent's age, education, occupation, income, and marital status (Table 1). The questionnaires were completed by 4517 parents of first-formers, response rate $-73.2 \%$.

\section{Statistical analysis}

Statistical analysis was performed by using SPSS 20.0 software for Windows. Descriptive statistics was applied. Children's nutrition habits were analyzed and compared according to their age and sex. Correlation among variables was evaluated by $\chi^{2}$. Links among nutrition habits of first-formers and family socioeconomic status were determined using binary logistic regression to calculate odds ratios (OR) and 95\% confidence intervals (CI). For all tests $\mathrm{p}<0.05$ was considered significant.

\section{Variables}

\section{Dependent variables}

Parents were asked to indicate the frequency of their children eating breakfast, fresh fruit and vegetables, softdrinks with sugar consumption by ticking one of the following five responses as it was mentioned above. Response options were recoded into dichotomous outcome variables $(1=$ daily; $0=$ less then daily). French fries, burgers, pizzas consumption were also recoded into dichotomous outcome variables $(1=$ daily or $4-6$ times a week; $0=$ less then 4 times a week).

\section{Independent variables}

Five socio-economic status variables (parent's age, marital status, education, occupation and income) were used in the analyses. Parental age was recoded into three categories (younger than 29 years; from 30 to 39; 40 year and older). Parental occupation from five possible response options was recoded into three categories (low completed high school; medium - completed college; high - completed graduate degree). Parental occupation from eight response options was recoded into three categories (work in state institution; work in non-state institution; unemployed). Family income from five response option was recoded into three categories (low - 400-600 Lt; middle - 600-1200 Lt and high >1200 Lt per month), later during binary logistic regression calculation family income from three option was recoded into two categories (low $<1200 \mathrm{Lt}$ and high $>1200 \mathrm{Lt}$ ) as middle income were merged with low income group.

\section{Results}

It was established that the majority (76\%) of Lithuanian first-formers eat breakfast every day or 4-6 times a week. Significant differences were found between breakfast consumption and gender - girls eat breakfast less frequently than boys. Fresh fruit and vegetable consumption is very important determinant regarding healthy lifestyle. While analyzing the data significant differences 
Table 1 Frequencies of first-formers food consumption

\begin{tabular}{|c|c|c|c|c|c|}
\hline & \multicolumn{2}{|c|}{ Children age } & \multicolumn{2}{|l|}{ Gender } & \multirow[t]{3}{*}{ Total \% (n) } \\
\hline & 7 & 8 & Boys & Girls & \\
\hline & $\%(n)$ & $\%(n)$ & $\%(n)$ & $\%(n)$ & \\
\hline \multicolumn{6}{|l|}{ Breakfast consumption } \\
\hline Every day and 4-6 times a week & $76.2(2386)$ & 75.4(1020) & $77.6(1713)$ & $74.4(1693)$ & $76(3406)$ \\
\hline 1-3 times a week and never & 23.8(746) & 24.6(332) & 22.4(495) & $25.6(583)$ & 24(1078) \\
\hline Total & $100(3132)$ & $100(1352)$ & 100 (2208) & $100(2276)$ & $100(4484)$ \\
\hline$x^{2} \mathrm{P}^{*}$ & - & & 0.012 & & \\
\hline \multicolumn{6}{|l|}{ Fresh fruits consumption } \\
\hline Every day and 4-6 times a week & $62.9(1934)$ & $57.5(762)$ & $59.9(1303)$ & $62.6(1393)$ & $61.3(2696)$ \\
\hline 1-3 times a week and never & $37.1(1140)$ & $42.5(564)$ & $40.1(872)$ & $37.4(832)$ & $38.7(1704)$ \\
\hline Total & $100(3102)$ & $100(1341)$ & $100(2275)$ & $100(2225)$ & $100(4400)$ \\
\hline$x^{2} \mathrm{P}^{*}$ & 0.001 & & - & & \\
\hline \multicolumn{6}{|l|}{ Fresh vegetables consumption } \\
\hline Every day and 4-6 times a week & $40.3(1183)$ & $40.8(525)$ & $39.7(822)$ & $41.1(886)$ & $40.4(1708)$ \\
\hline 1-3 times a week and never & $59.7(1755)$ & $59.2(763)$ & $60.3(1247)$ & $58.9(1271)$ & $59.6(2518)$ \\
\hline Total & 100 (2938) & $100(1288)$ & $100(2069)$ & $100(2157)$ & $100(4226)$ \\
\hline$x^{2} \mathrm{P}^{*}$ & - & & - & & \\
\hline \multicolumn{6}{|c|}{ Soft drinks with sugar consumption } \\
\hline Every day and 4-6 times a week & 15.9(373) & $17.2(178)$ & $16.7(285)$ & $15.9(266)$ & $16.3(551)$ \\
\hline 1-3 times a week and never & $84.1(1976)$ & $82.8(857)$ & $83.3(1423)$ & $84.1(1410)$ & $83.7(2833)$ \\
\hline Total & $100(2349)$ & $100(1035)$ & 100 (1708) & $100(1676)$ & $100(3384)$ \\
\hline$x^{2} \mathrm{P}^{*}$ & - & & - & & \\
\hline \multicolumn{6}{|c|}{ French fries, burgers, pizzas consumption } \\
\hline Every day and 4-6 times a week & $5(130)$ & $6.1(67)$ & $5.5(104)$ & $5(93)$ & $5.3(197)$ \\
\hline 1-3 times a week and never & $95(2488)$ & $93.9(1040)$ & $94.5(1773)$ & $95(1755)$ & $94.7(3528)$ \\
\hline Total & $100(2618)$ & $100(1107)$ & $100(1877)$ & $100(1848)$ & $100(3725)$ \\
\hline$x^{2} \mathrm{P}^{*}$ & - & & - & & \\
\hline
\end{tabular}

were found among first-formers age and fresh fruit consumption: higher percent of 7 year old children consumed fruits every day in comparison with 8 year olds. No differences were observed among gender of respondents and fresh fruit as well as vegetable consumption (Table 1). Unhealthy diet products (soft-drinks with sugar, French fries, burgers, pizzas) were consumed rarely: most of first-formers (from $82.8 \%$ to 95\%) eat these products 1-3 times a week or never and no significant differences were observed between gender and age.

The analysis of family socio-economic factors revealed that significant differences were found between parental age and their education, occupation, family income and marital status. Almost half of parents $(45.3 \%$ of fathers and $46.7 \%$ of mothers respectively) reported about completed high school and one-third (35.8\% of fathers and $34.4 \%$ of mothers respectively) of parents had completed graduated degree. Low educated fathers and mothers were mainly younger than 29 years old $(65.5 \%$ and $68.1 \%$ respectively). Generally $73.2 \%$ of fathers and $66.8 \%$ of mothers were employed, males - more frequently in private sector, females - in state institutions. Analysing by age it was determined that unemployment was more frequent in the group of the youngest parents, especially among women. More than half of respondents indicated low, one third - average and one in ten - high family income. The majority of children lived in two parent family (Table 2).

Logistic regression analysis revealed that mothers' and fathers' age had different influence on children habitual food consumption. Odds ratio of children daily breakfast consumption were 1.3 times higher in families where fathers' were older than 30 years comparing with younger fathers. Meanwhile mothers' age had significant influence just on children daily soft drinks with sugar consumption. Odds ratio of daily soft drinks with sugar consumption was 1.22 times smaller in families where 
Table 2 Socio-economic characteristics of families

\begin{tabular}{|c|c|c|c|c|c|c|c|c|}
\hline \multicolumn{7}{|l|}{ Parents' age } & \multirow{2}{*}{\multicolumn{2}{|c|}{ Gender }} \\
\hline & \multicolumn{2}{|l|}{ Mothers } & \multirow{3}{*}{40 yr. > more \% (n) } & \multicolumn{3}{|l|}{ Fathers } & & \\
\hline & $<29$ yr. & $30-39 \mathrm{yr}$. & & $<29$ yr. & 30-39 yr. & 40 yr. $>$ more $\%(n)$ & Males & Females \\
\hline & $\%(n)$ & $\%(n)$ & & $\%(n)$ & $\%(n)$ & & $\%(n)$ & $\%(n)$ \\
\hline \multicolumn{9}{|l|}{ Education } \\
\hline Low (completed high school) & $68.1(584)$ & $43.5(1158)$ & $35.2(298)$ & $65.5(205)$ & $45.1(1169)$ & $45.3(1857)$ & $45.3(1857)$ & $46.7(2041)$ \\
\hline Medium (completed college) & $10.1(87)$ & $18.7(497)$ & $28.3(239)$ & $11.5(36)$ & $17.9(464)$ & $23.00(275)$ & $18.9(775)$ & $18.8(823)$ \\
\hline High (completed graduate degree) & $21.8(187)$ & $37.8(1007)$ & 36.5 (309) & $23.00(72)$ & $37.00(960)$ & $36.5(436)$ & $35.8(1468)$ & $34.4(1503)$ \\
\hline Total & $100(858)$ & $100(2663)$ & $100(846)$ & $100(313)$ & $100(2593)$ & $100(1194)$ & $100(4100)$ & $100(4367)$ \\
\hline$x^{2}$ value & \multicolumn{3}{|c|}{$X 2=237.389 ; d f=4 ; p<0.001$} & \multicolumn{3}{|c|}{$X 2=71.211 ; d f=4 ; p<0.001$} & & \\
\hline \multicolumn{9}{|l|}{ Family income } \\
\hline Low (400-600 Lt) & $66.00(538)$ & $55.1(1401)$ & $56.7(459)$ & $69.8(210)$ & $54.9(1367)$ & $55.8(632)$ & $57.6(2398)$ & $56.3(2209)$ \\
\hline Middle (600-1200 Lt) & $27.5(224)$ & $31.4(798)$ & $33.6(272)$ & $25.2(76)$ & $32.2(802)$ & $32.9(372)$ & $31.1(1294)$ & $39.2(1746)$ \\
\hline High (>1200 Lt) & $6.5(53)$ & $13.5(342)$ & $9.8(79)$ & $5.00(15)$ & $12.8(319)$ & $11.3(128)$ & $11.4(474)$ & $11.8(462)$ \\
\hline Total & $100(815)$ & $100(2541)$ & $100(810)$ & $100(301)$ & $100(2566)$ & $100(1132)$ & $100(4166)$ & $100(3921)$ \\
\hline$x^{2}$ value & \multicolumn{3}{|c|}{$x^{2}=46.506 ; d f=4 ; p<0.001$} & \multicolumn{3}{|c|}{$x 2=29.444 ; d f=4 ; p<0.001$} & & \\
\hline \multicolumn{9}{|l|}{ Occupation } \\
\hline Work in state institutions & $13.9(122)$ & $29.3(796)$ & $36.3(311)$ & $9.1(29)$ & $18.3(487)$ & $22.6(274)$ & $18.9(790)$ & $27.6(1229)$ \\
\hline Work in private sector & $39.7(349)$ & $40.8(1109)$ & $33.6(288)$ & $50.9(163)$ & $57.3(1523)$ & $48.4(587)$ & $54.3(2273)$ & $39.2(1746)$ \\
\hline Unemployed & $46.4(408)$ & $29.9(814)$ & $30.00(257)$ & $40.00(129)$ & $24.3(646)$ & $29.00(352)$ & $26.8(1126)$ & $33.2(1479)$ \\
\hline Total & $100(581)$ & $100(2719)$ & $100(856)$ & $100(320)$ & $100(2656)$ & $100(1213)$ & 100 (4189) & $100(4454)$ \\
\hline$x^{2}$ value & \multicolumn{3}{|c|}{$x 2=125.486 ; d f=4 ; p<0.001$} & \multicolumn{3}{|c|}{$X 2=67.634 ; d f=4 ; p<0.001$} & & \\
\hline \multicolumn{9}{|l|}{ Marital status } \\
\hline Married & & & & & & & $84.8(3492)$ & $80.3(3505)$ \\
\hline Single, divorced, other & & & & & & & $15.2(627)$ & $19.7(860)$ \\
\hline Total & & & & & & & 100 (4119) & $100(4365)$ \\
\hline$x^{2}$ value & & & & & & & & \\
\hline
\end{tabular}

$\mathrm{P}^{*}$ - significance comparing groups (Chi-squares test). 
mothers' were older than 30 years comparing with younger ones. The analysis established that mothers' age had no significant impact for children breakfast and fresh fruit consumption, contrary to parental education. It was determined that odds ratio of every day fresh fruit consumption was 1.3 and 1.4 higher of those children whose mothers and fathers had completed graduate degree comparing with low educated parents. Also it was determined that odds ratio of children every day fresh fruit consumption 1.3 and 1.4 higher of those children whose mothers and fathers had completed graduate degree comparing with low educated parents. High parental education had influence on first-formers daily soft drinks with sugar consumption. Odds ratio of soft drinks with sugar consumption was 2.6 and 1.7 times lower among first-formers whose mothers and fathers had high education compared with low educated parents (Table 3). Logistic regression analysis established that family income and marital status had significant impact for children's daily fruit and soft drinks with sugar consumption, but no impact for daily breakfast consumption. Odds ratio of children fresh fruit consumption was 1.5 times higher in families with high income comparing with low income families. Odds ratio of first-formers soft beverage with sugar consumption was 1.6 and 1.3 times lower in full or high income families compared with single parent, low income families (Table 4).

\section{Discussion}

Breakfast consumption is very important for children future health [18-22]. Epidemiological research claims that despite the potential importance of breakfast consumption, the prevalence rates of breakfast skipping among children and adolescents has increased in the past few decades $[18,22,23]$. Current study findings indicate that $76 \%$ of Lithuanian first-formers eat breakfast every day, girls less frequently than boys. Our national data results exhibit lower prevalence of daily breakfast than the average rates of the same COSI study of Portuguese primary school age children. It is reported that in $201095.1 \%$ of 6-8 year old Portuguese children have breakfast every day [24]. But our results about having breakfast every day were significantly better than the results of Western Sicilia primary school children. The study conducted in Italy when $8-11$ year old children were examined, revealed that only $54 \%$ of respondents have breakfast every day [25]. Comparing our data with the results of other national survey in Lithuania, primary school-age children eat breakfast more frequently than 11-year olds from Health Behaviour in School-Aged Children (HBSC) study performed in 2010, which results show that $65 \%$ of teenagers eat breakfast every [26]. The differences between the results of these two national studies' can probably be explained by stronger parental attention and influence for first-formers eating patterns and different age of respondents. Many international studies confirm that the prevalence of regular breakfast consumption tends to decrease as children grow older $[18,27,28]$. Another important children's eating behaviour component is consuming a diet high in fruits and vegetables which is associated with lower risks of numerous chronic

Table 3 Odds Ratios of children daily breakfast, fresh fruit and soft drinks with sugar consumption by parents' age and education

\begin{tabular}{|c|c|c|c|c|c|c|}
\hline & \multicolumn{2}{|c|}{ Daily breakfast consumption } & \multicolumn{2}{|c|}{ Daily fresh fruit consumption } & \multicolumn{2}{|c|}{ Daily soft drinks with sugar consumption } \\
\hline & Proportion \% & OR $(95 \% \mathrm{Cl})$ & Proportion \% & OR $(95 \% \mathrm{Cl})$ & Proportion \% & OR $(95 \% \mathrm{Cl})$ \\
\hline \multicolumn{7}{|l|}{ Age females (mothers) } \\
\hline$<29 \mathrm{yr}$ & $70.4 \%$ & 1 & $56.2 \%$ & 1 & $21.7 \%$ & 1 \\
\hline $30-39$ yr. & $76.6 \%$ & $1.135(0.974 ; 1.323)$ & $61.1 \%$ & $1.097(0.955 ; 1.259)$ & $14.7 \%$ & $0.823(0.689 ; 0.984)$ \\
\hline $40 \mathrm{yr} .>$ more & $77.4 \%$ & $1.122(0 ; 898$ 1.402) & $61.8 \%$ & $1.051(0.863 ; 1.280)$ & $13.4 \%$ & $0.817(0.624 ; 1.070)$ \\
\hline \multicolumn{7}{|l|}{ Age males (fathers) } \\
\hline$<29 \mathrm{yr}$ & $68.7 \%$ & 1 & $55.1 \%$ & 1 & $21.6 \%$ & 1 \\
\hline 30-39 yr. & $75.8 \%$ & $1.267(1.027 ; 1.564)$ & $60.5 \%$ & $1.071(0.88 ; 1.303)$ & $15.3 \%$ & $0.878(0.688 ; 1.120)$ \\
\hline $40 \mathrm{yr} .>$ more & $77.3 \%$ & $1.291(1.004 ; 1.661)$ & $62.0 \%$ & $1.112(0.884 ; 1.400)$ & $13.8 \%$ & $0.864(0.641 ; 1.163)$ \\
\hline \multicolumn{7}{|l|}{ Education fathers } \\
\hline Low (completed high school) & $73.1 \%$ & 1 & $57.1 \%$ & 1 & $19.2 \%$ & 1 \\
\hline Medium (completed college) & $76.2 \%$ & $1.066(0.927 ; 1.266)$ & $61.6 \%$ & $1.097(0.97 ; 1.241)$ & $13.7 \%$ & $0.892(0.752 ; 1.058)$ \\
\hline High (completed graduate degree) & $82.6 \%$ & $1.440(1.220 ; 1.700)$ & $69.1 \%$ & $1.369(1.189 ; 1.576)$ & $7.2 \%$ & $0.581(0.461 ; 0.731)$ \\
\hline \multicolumn{7}{|l|}{ Education mothers } \\
\hline Low (completed high school) & $72.0 \%$ & 1 & $55.5 \%$ & 1 & $22.6 \%$ & 1 \\
\hline Medium (completed college) & $76.3 \%$ & $1.130(0.976 ; 1.309)$ & $61.9 \%$ & $1.170(1.028 ; 1.331)$ & $13.4 \%$ & $0.616(0.516 ; 0.737)$ \\
\hline High (completed graduate degree) & $80.5 \%$ & $1.293(1.119 ; 1.492)$ & $66.7 \%$ & $1.338(1.224 ; 1.573)$ & $7.9 \%$ & $0.392(0.229 ; 0.669)$ \\
\hline
\end{tabular}


Table 4 Odds Ratios of children daily breakfast, fresh fruit and soft drinks with sugar consumption by family income and marital status

\begin{tabular}{|c|c|c|c|c|c|c|}
\hline & \multicolumn{2}{|c|}{ Daily breakfast consumption } & \multicolumn{2}{|c|}{ Daily fresh fruit consumption } & \multicolumn{2}{|c|}{ Daily soft drinks with sugar consumption } \\
\hline & Proportion \% & OR $(95 \% \mathrm{Cl})$ & Proportion \% & OR $(95 \% \mathrm{Cl})$ & Proportion \% & OR $(95 \% \mathrm{Cl})$ \\
\hline \multicolumn{7}{|l|}{ Family income } \\
\hline Low $(<1200$ Lt) & $75.0 \%$ & 1 & $58.6 \%$ & 1 & $16.8 \%$ & 1 \\
\hline High (>1200 Lt) & $77.9 \%$ & $1.142(0.978 ; 1.334)$ & $68.7 \%$ & $1.547(1.346 ; 1.779)$ & $11.0 \%$ & $0.636(0.518 ; 0.780)$ \\
\hline \multicolumn{7}{|l|}{ Marital status } \\
\hline Divorced & $73.7 \%$ & 1 & $57.3 \%$ & 1 & $19.5 \%$ & 1 \\
\hline Married & $75.9 \%$ & $1.110(0.979 ; 1.259)$ & $60.90 \%$ & $1.086(0.970 ; 1.216)$ & & $0.772(0.668 ; 0.892)$ \\
\hline
\end{tabular}

diseases. According to our study results daily intake of fresh fruit and vegetable is $61.3 \%$ and $40.4 \%$ respectively. Our study results on children fresh fruit and vegetable consumption rates differ from HBSC (2010) study. Daily intake of fresh fruit is twofold lower by 11 year olds (30\%) but daily vegetable consumption is 1.4 times higher (54.5\%) [26]. Such differences can be explained by the fact that primary school age children more often eat at home, they prefer to eat fruits more than vegetables, as fruits are usually sweet, juicy, and can be eaten fresh, and adolescents are more influenced by friends, social advertisements and willingness to try new snacks, drinks, instead of eating fruits, but perhaps vegetable salads as snacks are also more popular among older children.

Parents play a major role in the development of healthy eating habits of their children through a variety of mechanisms including a healthy diet, the availability and accessibility of nutritious foods at home, and the development of attitudes, values, preferences and role modelling [22-24]. The main findings of this study confirm links between first-formers nutrition habits and family socio-economic status in Lithuania. Parent's income is one of the primary indicators of socio-economic status. Our results show that parent's higher education and income have significant positive impact on daily breakfast, fresh fruit consumption of first-formers compared with lower socio-economic position families. In other studies family income is also mentioned as factor linked with higher intake of fruit and vegetables among children [22,23]. The similar outcome presents HBSC study. In the international report on social determinants of health and well-being among young people it is stated that adolescents from high-affluence families in most countries and regions were significantly more likely to report eating breakfast, fresh fruit daily [26]. Current study findings indicate that odds ratio of children soft drinks with sugar consumption were lower in families with high income comparing with low income families. Many studies from different countries confirm these links and claim that prevalence of children and adolescents' unhealthy behavior including diet tend to be higher in families with low socio-economic position compared with high social class households [19,29-35]. Interesting results of soft-drinks consumption are presented by CA Vereecken at all. It is established that soft drinks consumption is lower among pupils of higher parental occupational status in Northern, Southern and Western European countries, but not in Central and Eastern European countries. Analysing the HBSC 2001/2002 survey results the authors stated that only in Central and Eastern European countries is observed significant increase in soft drink consumption with increasing family affluence [36]. We can state that Lithuanian situation has changed during more than ten year period and there are no differences from Western European countries.

\section{Strengths and limitations}

Among the strengths of the present study this is the first study to assess first-formers nutritional habits and 4 socio-economic aspects of family (education, occupation, family income and marital status. The present study has some limitations. Firstly this study has a cross-sectional design, which does not allow to draw conclusions about causal relationships. Secondly, assessments of child's habitual food consumption were based on parent's self-reports which cannot exclude misreporting, recall biases.

\section{Conclusions}

Results from the national survey of primary school age children of Lithuania reveals that family socio-economic position plays one of the major role in breakfast, fresh fruit and soft drinks with sugar consumption among younger school age children. Likelihood of daily breakfast, and fresh fruit consumption of first-formers were higher in high socio-economic status families compared with low. Opposite results were found in soft drinks with sugar consumption. This social patterning should be recognized in public health interventions. 


\section{Competing interests}

The authors declare that they have no competing interests.

\section{Authors' contributions}

AP is the main coordinator of COSI study in Lithuania. VŽ conducted the analyses, interpreted the data and drafted the manuscript. VŽ, AP and EA advised on data analysis and interpretation of results. All authors critically revised the manuscript and approved of it in its final form.

Received: 3 December 2014 Accepted: 13 April 2015

Published online: 23 April 2015

\section{References}

1. Preedy VR, Hunter L-A, Patel VB. Diet quality: an evidence-based approach. Springer Sci Bus Media. 2013;1:340.

2. WHO. Diet, nutrition and the prevention of chronic diseases. Report of the joint WHO/FAO expert consultation. http://www.who.int/dietphysicalactivity/ publications/trs916/download/en/. Accessed 14 Nov 2014.

3. Cooke L. The importance of exposure for healthy eating in childhood: a review. J Hum Nutr Diet. 2007;20:294-301.

4. Cutler G, Flood A, Hannan P, Neumark-Sztainer D. Multiple sociodemographic and socio environmental characteristics are correlated with major patterns of dietary intake in adolescents. J Am Diet Assoc. 2011;111:230-40.

5. Patrick $H$, Nicklas T. A review of family and social determinants of children's eating patterns and diet quality. J Am Coll Nutr. 2005;24:83-92.

6. Nyberg G, Sundblom E, Norman A, Elinder LS. A healthy school startparental support to promote healthy dietary habits and physical activity in children: design and evaluation of a cluster-randomised intervention. BMC Public Health. 2011;11:185.

7. Cullen KW, Ash DM, Warneke C, de Moor C. Intake of soft drinks, fruitflavored beverages, and fruits and vegetables by children in grades 4 through 6. Am J Public Health. 2002;92(9):1475-8.

8. Darmon N, Drewnowski A. Does social class predict diet quality? Am J Clin Nutr. 2008:87:1107-17.

9. Nelson M. Childhood nutrition and poverty. Proc Nutr Soc. 2000;59(2):307-15.

10. Hoglund D, Samuelson G, Mark A. Food habits in Swedish adolescents in relation to socioeconomic conditions. Eur J Clin Nutr. 1998;52(11):784-9.

11. Mathieson A, Koller T. Addressing the socioeconomic determinants of healthy eating habits and physical activity levels among adolescents. WHO/ HBSC FORUM 2006. Copenhagen: World Health Organization; 2006.

12. Ramic E, Durakovic S, Karic E, Mujanovic O, Alibasic E. Influence of lifestyle on overweight and obesity in school-age children. Med Arch. 2009;63:284-7.

13. Li J, Hooker N. Childhood obesity and schools: evidence from the national survey of children's health. J Sch Health. 2010;80:96-103.

14. Skafida V, Treanor MC. Do changes in objective and subjective family income predict change in children's diets over time? unique insights using a longitudinal cohort study and fixed effects analysis. J Epidemiol Community Health. 2014;68:534-41.

15. Drewnowski A, Darmon N. Food choices and diet costs: an economic analysis. J Nutr. 2005;135:900-4.

16. World Health Organization Regional Office for Europe. The new European policy for health - Health 2020 Policy framework and strategy. Draft 2 Denmark: WHO; 2012.

17. Zaborskis A, Lagunaite R, Busha R, Lubiene J, et al. Trend in eating habits among Lithuanian school-aged children in context of social inequality: three cross-sectional surveys 2002, 2006 and 2010. BMC Public Health. 2012;12:52.

18. Affenito SG. Breakfast: a missed opportunity. J Am Diet Assoc. 2007;107:565-9.

19. Haug E, Rasmussen M, Samdal O, lannotti R, Kelly C, Borraccino A, et al. Overweight in school-aged children and its relation with demographic and lifestyle factors: results from the WHO-Collaborative Health Behaviour in School-aged Children (HBSC) Study. Int J Public Health. 2009;54(S2):167-79.

20. Keski-Rahkonen A, Kaprio J, Rissanen A, Virkkunen M, Rose RJ. Breakfast skipping and health-compromising behaviors in adolescents and adults. Eur J Clin Nutr. 2003;57:842-53.

21. Rampersaud GC, Pereira MA, Girard BL, Adams J, Metzl JD. Review breakfast habits, nutritional status, body weight, and academic performance in children and adolescents. J Am Diet Assoc. 2005:105:743-60.

22. Deshmukh-Taskar PR, Nicklas TA, O'Neil CE, Keast DR, Radcliffe JD, Cho S. The relationship of breakfast skipping and type of breakfast consumption with nutrient intake and weight status in children and adolescents: the national health and nutrition examination survey. 1999-2006. J Am Diet Assoc. 2010;110(6):869-78.

23. Timlin MT, Pereira MA, Story M, Neumark-Sztainer D. Breakfast eating and weight change in a 5-year prospective analysis of adolescents: Project EAT (eating among teens). Pediatrics. 2008;121(3):E638-45.

24. Duarte D, Ramos C, Carvalho MA, Rito A. Breakfast consumption in primary school children from cosi Portugal 2010 study. http://hdl.handle.net/ 10400.18/2095. Accessed 17 Nov 2014.

25. Enza S, Patrizia M, Gabriele P, Giuseppe T. Body Mass Index, family lifestyle, physical activity and eating behavior on a sample of primary school students in a small town of Western Sicily. JPH. 2009:6(3):205-17.

26. Currie C, Zanotti C, Morgan A, Currie D, de Looze M, Roberts C, et al. Social determinants of health and well-being among young people. Health behaviour in school-aged Children (HBSC) study: international report from the 2009/2010 survey. Copenhagen: WHO Regional Office for Europe; 2012. Health Policy for children and Adolescents, No 6.

27. Petersen PE, Kwan S. The 7th WHO global conference on health promotion - towards integration of oral health. Community Dent Health. 2010;27(1):129-36.

28. Levin KA, Currie C. Adolescent tooth brushing and the home environment sociodemographic factors, family relationships and mealtime routines and disorganization. Community Dent Oral Epidemiol. 2010;38(1):10-8.

29. Taylor JP, Evers S, McKenna M. Determinants of healthy eating in children and youth. Can J Public Health. 2005;96(3):S20-6, S22-29.

30. Kirkpatrick $S$, Tarasuk $V$. The relationship between low income and household food expenditure patterns in Canada. Public Health Nutr. 2003;6:589-97.

31. Riediger ND, Shooshtari S, Moghadasian MH. The influence of sociodemographic factors on patterns of fruit and vegetable consumption in Canadian adolescents. J Am Diet Assoc. 2007;107:1511-8.

32. Turrell G, Hewitt B, Patterson C, Oldenburg B, Gould T. Socioeconomic differences in food purchasing behaviour and suggested implications for diet-related health promotion. J Hum Nutr Diet. 2002;15(5):355-64.

33. Drewnowski A, Specter SE. Poverty and obesity: the role of energy density and energy costs. Am J Clin Nutr. 2004;79(1):6-16.

34. De Coen V, Vansteelandt S, Maes L, Huybrechts I, De Bourdeaudhuij I, Vereecken C. Parental socioeconomic status and soft drink consumption of the child. The mediating proportion of parenting practices. Appetite. 2012;59(1):76-80.

35. Skardal M, Western IM, Ask AMS, Øverby NC. Socioeconomic differences in selected dietary habits among Norwegian 13-14 year-olds: a cross-sectional study. Food Nutr Res. 2014;58:23590.

36. Vereecken CA, Inchley J, Subramanian SV, Hublet A, Maes L. The relative influence of individual and contextual socio-economic status on concumption of fruit and soft drinks among adolescents in Europe. Eur J Public Health. 2005;15(3):224-32.

\section{Submit your next manuscript to BioMed Central and take full advantage of:}

- Convenient online submission

- Thorough peer review

- No space constraints or color figure charges

- Immediate publication on acceptance

- Inclusion in PubMed, CAS, Scopus and Google Scholar

- Research which is freely available for redistribution 